\title{
EDITORIAL
}

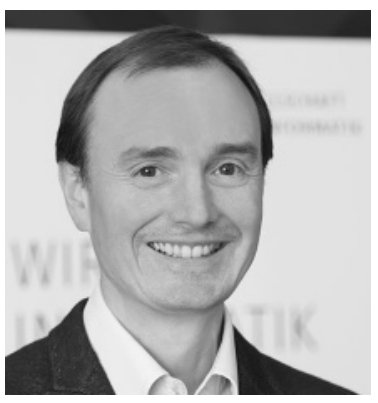

\author{
Hannes Federrath, \\ Präsident der GI, \\ Universität Hamburg, \\ E-Mail: \\ federrath@informatik.uni-hamburg.de
}

\section{Wie soll ich arbeiten? Was soll ich tun?}

Hätten Informatikerinnen und Informatiker gern etwas mit dem Eid des Hippokrates Vergleichbares? Wenngleich eine mehr als 2000 Jahre alte ärztliche Ethik keinerlei direkte Rechtswirkung entfaltet, so gehen aus ihr heute rechtlich gültige Prinzipien wie etwa die ärztliche Schweigepflicht und das ausschließliche Handeln zum Wohle des Patienten hervor.

Gesundheits-Apps können inzwischen recht zuverlässig Diagnosen zu weit verbreiteten Symptomen stellen. Sie nutzen Massendaten, die (auch) in den Händen von Nichtmedizinern sind. Während das Arzt-Patienten-Verhältnis geschützt ist, gilt dies nicht für das Patienten-Gesundheits-App-Programmierer-Verhältnis.

Für Informatikerinnen und Informatiker stellen sich mehr und mehr Fragen nach dem richtigen Handeln diesseits und jenseits der rechtlich festgeschriebenen Regeln: „Was schützt einen Patienten vor dem Missbrauch seiner Daten durch den Gesundheits-App-Programmierer?"

Wenn ich als Patient meine Symptome verheimliche, wird eine Gesundheits-App wohl kaum eine Diagnose stellen können. Naja, wer weiß ... Unsere Smartphones verfolgen uns ja praktisch in jeder Lebenslage.

Big Data, Data Science und Kl-Forscher lassen uns wissen, dass Daten Gold wert sind. Ein junger Informatiker und Berufseinsteiger könnte sich fragen: „Soll ich diese Funktion zur Überwachung der Nutzer wirklich so in die Software einbauen? Eigentlich müsste darüber in der Datenschutzerklärung informiert werden, aber das scheint hier niemand zu wissen oder zu wollen." Oder: "Ist eine solche Abschalteinrichtung alleine deshalb vertretbar, weil es doch fast alle so machen?"

Nicht selten stellt sich den Systementwicklern auch die Frage des Dual-Use: „Dieses Bildverarbeitungsmodul zur Steuerung eines autonomen Systems kann Menschenleben retten. Kann ich es - und ggf. wie - so gestalten, dass es nur für zivile Einsatzzwecke geeignet ist?" Die Digitalisierung rückt die Verantwortung unseres Berufstandes bei der Gestaltung von Technik und Gesellschaft mehr und mehr in den Mittelpunkt.

Die Gesellschaft für Informatik hat immerhin seit 25 Jahren ethische Leitlinien. 2018 wurden sie, nachdem sie 2004 schon einmal überarbeitet wurden, an die veränderten gesellschaftlichen und technischen Rahmenbedingungen angepasst. Im Beitrag „Die neuen ethischen Leitlinien der GI" finden Sie den Text der Leitlinien sowie einige der Herausforderungen und Rahmenbedingungen, unter denen die neuen Leitlinien entstanden sind. Das Präsidium der GI hat die ethischen Leitlinien im Juni 2018 einstimmig verabschiedet.

Dieses Jahr feiert die Gl ihr 50-jähriges Bestehen. Da kommt der Beitrag von Wolfgang Coy zur "Geschichte der ethischen Leitlinien" gerade recht, um herauszuarbeiten, dass Informatiker im Ringen um unsere gesellschaftliche Verantwortung - genauso wie Physiker und Mediziner - wohl niemals fertig sein werden, wenngleich dieser Prozess bei den älteren Disziplinen weitaus länger dauern dürfte und vermutlich deutlich schmerzhafter war (denken wir nur an Selbstversuche von Wissenschaftlern und den Bau von Atomwaffen) als bei Informatikern.

Coy beschreibt in seinem Beitrag auch kurz die Entstehungsgeschichte der "Gewissensbits". Die Auseinandersetzung zu Fragen der Verantwortung der Informatikerin und des Informatikers wird seit nunmehr zehn Jahren auch im Informatik-Spektrum motiviert. Christina B. Class, Constanze Kurz und Debora Weber-Wulff geben in ihrem Beitrag einen Abriss über die methodischen und pädagogischen Ideen dieses Formats. Die Gewissensbits hätten all die Jahre auch als Titel "Keine eindeutige Antwort" tragen können. Mein persönlicher Wunsch wäre es oft gewesen, wenigstens eine der möglichen Antworten von den Fachleuten zu bekommen - vielleicht eine Anregung für die Zukunft?

https://doi.org/10.1007/s00287-019-01156-3 


\section{\{ EDITORIAL}

Ranga Yogeshwar bezeichnet die Digitalisierung als „Kultur des Neuen“ und plädiert für eine kritische Betrachtung der Risiken und einen optimistischen Blick nach vorn. Der Chefredakteur der Informatik-Spektrum, Peter Pagel, hat mit ihm ein sehr lesenswertes Interview geführt. Eine Begebenheit, die sich nicht in dem Interview wiederfindet, möchte ich Ihnen nicht vorenthalten: Ranga Yogeshwar hatte in seinem brillanten Eröffnungsvortrag auf der Jahrestagung INFORMATIK 2018 in Berlin dem Publikum zwei Klavierstücke dargeboten; eines wurde von einer KI gespielt, ein weiteres von seiner Tochter. Dem Publikum fiel es extrem schwer, eine Entscheidung zu treffen, welches Stück vom Menschen und welches von der Maschine stammte. Willkommen in der Zukunft.

Künstliche Intelligenz und Informatik sind zwar nicht dasselbe, wenngleich man derzeit den Eindruck bekommen könnte, dass Informatik sehr verkürzt auf KI reduziert wird. Wir Informatikerinnen und Informatiker werden aber auch weiterhin Software, Systeme und auch programmierbare Hardware benötigen und diese verantwortungsvoll und vorausschauend entwickeln. Dies schließt die KI selbstverständlich mit ein. Für die zahlreichen Diskussionsbeiträge im Vorfeld der Novellierung der ethischen Leitlinien danke ich Ihnen ganz herzlich und freue mich, wenn Sie den Diskussionsprozess weiter begleiten. 


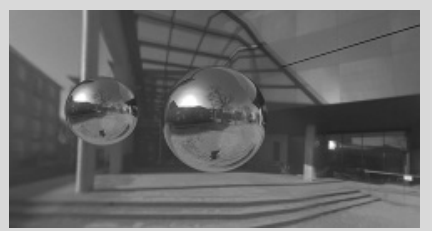

Das Cover-Bild zeigt den Haupteingang des (relativ) neuen R-Gebäudes der Hochschule München mit dem roten Hörsaal (sog. „roter Würfel"), der auch als Symbol für das Logo der Hochschule München verwendet wurde. Außerdem sieht man noch zwei verspiegelte $\mathrm{Ku}$ - geln, in denen sich die Umgebung spiegelt (allerdings spiegelt sich aufgrund des CubeMapping-Spiegelungsverfahrens nur die Umgebung in den Kugeln, nicht die Kugeln sich gegenseitig).

Das Programm zur Erzeugung des Bildes hat Prof. Dr. Alfred Nischwitz geschrieben. Es ist Teil einer Praktikumsaufgabe für Masterstudierende in seiner Lehrveranstaltung "Computergrafik" und demonstriert echtzeitfähige Spiegelungen in OpenGL (Cube-Mapping-Spiegelung). Die Fotos für die Cube Map (es wurden sechs Bilder der Umgebung auf einem Kubus nahtlos aufgebracht) hat ein früherer Student von Prof. Nischwitz, Alex Link, gemacht.

Kontakt:

Prof. Dr. Alfred Nischwitz

Computergrafik, Bildverarbeitung und Maschinelles Lernen,

Fakultät 07 für Informatik und Mathematik, Hochschule München/University of Applied Sciences,

München Lothstr. 64, 80335 München, E-Mail: nischwitz@lrz.fh-muenchen.de 